\title{
Diagnostic des facteurs limitants du rendement du blé dur en parcelles d'agriculteurs dans une petite région semi-aride en Tunisie
}

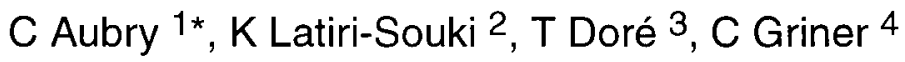 \\ IINRA, laboratoire SAD, F78850 Thiverval-Grignon; \\ 2 Centre de recherches du génie rural, BP 10, 2080 L'Ariana, Tunisie; \\ 3 INA-PG, chaire d'agronomie, 16, rue Claude-Bernard, F75005 Paris; \\ 4 SCA-Agriculteurs de Bretagne, Lanvinou, F29206 Landerneau, France
}

(Reçu le 16 novembre 1992; accepté le 2 mars 1994)

\begin{abstract}
Résumé - Dans un article précédent (Latiri-Souki et al, 1992), on a paramétré des relations entre composantes du rendement du blé dur en climat semi-aride, en l'absence de facteurs limitants. Ces relations ont servi à établir un diagnostic des facteurs et conditions limitants rencontrés dans un réseau de parcelles d'agriculteurs lors de 2 années climatiquement contrastées. Pour chaque scénario climatique, on obtient une forte variabilité des rendements entre parcelles, fonction du nombre de grains par unité de surface et essentiellement déterminée avant la floraison. La maîtrise de la levée, la nutrition azotée et l'infestation en adventices se sont avérées chaque année des facteurs majeurs de variation des rendements.
\end{abstract}

Triticum durum Desf / semi-aride / diagnostic / élaboration du rendement

Summary - Diagnosis of durum wheat yield in agricultural fields under semi-arid conditions in Tunisia. In order to determine the main limiting factors of wheat production in a semi-arid area of Tunisia, growth, yield, and yield components of durum wheat were measured in agricultural fields over 2 years with contrasting rainfall. They were compared with controls established under experimental conditions and published in a previous paper. Dry matter production, yield, and yield component were highly variable among and within a climatic year. Yield was mainly related to grain number for both years; grain and ear number variability depend on growth before anthesis. Comparison with the controls allowed us to determine the main periods when limiting factors applied. Poor seedbed control, weed infestation, nitrogen supply, and water availability during the growing season, were all identified as main limiting factors. Some of these are related to constraints from the cropping systems.

Triticum durum Desf / semi-arid conditions / diagnosis / yield components

\section{INTRODUCTION}

En Tunisie, le blé dur représente une part importante des 1,5 millions d'hectares (soit $30 \%$ des terres labourables) cultivés en céréales. En climat semi-aride, cette culture est présente dans la quasi-totalité des exploitations et sa production connaît de très fortes variations interannuelles que la plupart des auteurs relient à celles de la pluviométrie, particulièrement importantes dans cet étage climatique (El Amami et Bouzaïdi, 1979; Papy, 1979; Lelièvre et al, 1981).

\footnotetext{
* Correspondance et tirés à part.
} 
À cette variabilité interannuelle des rendements du blé dur se superposent, dans une région et pour un scénario climatique donnés, des variations intra-annuelles dont l'ampleur et les déterminants sont moins connus. Certaines études soulignent cependant l'influence d'éléments du milieu et de la conduite technique agissant en interaction, comme les dates de semis ou la fertilisation azotée (Lelièvre et al, 1981), la réussite des levées selon les conditions techniques des semis et le climat automnal (Fenech et Papy, 1977), le type de sol et les variétés (Collectif, 1988).

L'objectif du présent mémoire est d'identifier, dans une petite région de climat semi-aride en Tunisie, les facteurs et conditions limitant le rendement du blé dur qui sont à l'origine des variations de production constatées entre parcelles d'agriculteurs une année donnée.

Pour ce faire, on a opté pour une démarche de diagnostic régional au champ, mise au point sur le blé tendre en France puis extrapolée à d'autres cultures (Sebillotte et al, 1978 ; Meynard et Sebillotte, 1982 ; Manichon et al, 1989 ; Meynard et David, 1992). Cette démarche recourt de façon conjointe à l'expérimentation et à l'enquête au champ et comprend plusieurs étapes: i) dans une petite région où l'on peut considérer le climat comme homogène, on constitue un dispositif expérimental visant à obtenir, pour un cultivar donné, des modèles d'élaboration du rendement en l'absence de facteurs limitants ; ii) à partir d'enquêtes, on élabore des hypothèses sur les principaux facteurs du milieu et des techniques culturales à l'origine des variations de production dans la région et on construit un dispositif expérimental pour étudier l'effet de ces facteurs considérés a priori comme importants ; iii) on construit, sur plusieurs années culturales, un réseau de parcelles d'agriculteurs prospectant la gamme des types de sols de la région et des techniques pratiquées par les agriculteurs et on analyse les écarts de niveaux des composantes du rendement entre parcelles et références expérimentales en les reliant aux états du milieu, aux états du peuplement et aux techniques enregistrés sur les parcelles.

Cette démarche a été privilégiée par rapport à une démarche uniquement expérimentale, car elle permet de rendre compte de la variabilité des situations agricoles et de les resituer dans un référentiel local (Sebilotte, 1978, 1990). Elle a également été retenue face à une démarche qui consiste à établir, à partir de données recueillies en parcelles agricoles, des liaisons statistiques entre les rendements (ou leurs principales composantes) et les pratiques des agriculteurs ou les caractéristiques permanentes du milieu (Collectif 1988 ; Burleigh et al, 1991 ; Shafiq et al, 1993). Cette dernière méthode, dérivée des méthodes d'élaboration de modèles agroclimatiques et agrotechniques (voir par exemple Ollivier et Malet, 1979), ne permet généralement par d'attribuer de façon suffisamment certaine une cause à une variation observée. Enfin, la méthode de diagnostic régional au champ que nous avons retenue diffère de celle adoptée par Durrant (1988), laquelle consiste en une quantification en parcelles agricoles du poids respectif de chacun des facteurs de variation par phase d'élaboration du rendement, après recensement de tous ces facteurs (Durrant et al, 1984).

On a exposé dans un précédent mémoire (Latiri-Souki et al, 1992) les résultats des étapes i) et ii) de la démarche appliquée au blé dur dans la petite région semi-aride de Zaghouan. On rappellera dans ce mémoire les principaux résultats obtenus avant d'appliquer l'étape iii) à des réseaux de parcelles agricoles préalablement caractérisés.

\section{MATÉRIELS ET MÉTHODES}

Cette étude a eu lieu lors de 2 années présentant une pluviométrie contrastée : les précipitations mensuelles au cours de la première campagne (1984-1985) sont proches du $9^{e}$ décile (voire supérieures) tout au long du cycle du blé ; la seconde campagne (1985-1986) a été marquée par une sécheresse forte, les précipitations au cours du cycle se situant à proximité du $1^{\text {er }}$ décile, voire en dessous pour l'automne et le début de l'hiver.

\section{Rappel des références expérimentales (Latiri-Souki et al, 1992)}

On a paramétré [étape i)], pour la variété de blé dur Karim, des relations entre composantes du rendement en l'absence de facteurs limitants établies sur blé tendre en climat tempéré (Meynard, 1985a). Ce paramétrage (tableau I) a été effectué lors de la campagne culturale pluvieuse (1984-1985), dans un sol à forte réserve utile (Tell, sol argileux profond) et avec une conduite comprenant un contrôle total des adventices et une alimentation azotée pléthorique. Les relations faisant intervenir la biomasse aérienne au début de la montaison (relations 1 et $1 b$ ) sont peu utilisables du 
Tableau I. Rappel des références expérimentales (Latiri-Souki et al, 1992).

Phase du cycle

Relation entre composantes
Remarques
Levée à début montaison

Levée à épiaison

Début montaison à fécondation

Fécondation à maturité

Fécondation

Levée à récolte
(1) $\mathrm{BA} 1=0.47 * \mathrm{NP}$

$r^{2}=0,77 n=13$

(1b) $\mathrm{BA} 1=\mathrm{NP} /\left(1,91+4.3 * \mathrm{NP} * 10^{-3}\right)$ $r^{2}=0,41 n=17$

(2b) $\mathrm{NE}=52,5$ * NP0.35

$r^{2}=0,49 n=30$

(3) $\mathrm{NG}=27,2$ * $\mathrm{NE}+3759$

$r^{2}=0,78 n=30$

$\mathrm{R}=4,63^{*} 10^{-3}$ * $\mathrm{NG}$

$r^{2}=0,97 n=20$

(5) $\mathrm{NG}=16,6$ * $\mathrm{PP}$

$r^{2}=0,97 n=30$

(6) $\mathrm{QNR}=13,1{ }^{*} \mathrm{NG}{ }^{*} 10^{-3}$

$r^{2}=0,98 n=3$
$B A 1 \leq 70 \mathrm{~g} / \mathrm{m}^{2}$

$\mathrm{NP} \leq 160$ pieds $/ \mathrm{m}^{2}$

autres valeurs

toutes valeurs

de NE et NP

$\mathrm{P} 1 \mathrm{Gmax}=46.3 \mathrm{mg}$

$\mathrm{NP}=$ nombre de pieds par $\mathrm{m}^{2} ; \mathrm{BA} 1$ = biomasse aérienne au début de la montaison $\left(\mathrm{g} / \mathrm{m}^{2}\right) ; \mathrm{NE}=$ nombre d'épis par $\mathrm{m}^{2}: \mathrm{NG}=$ nombre de grains par $m^{2} ; R=$ rendement à $0 \%$ d'humidite $(q / h a) ; P 1 G m a x=$ poids moyen d'un grain maximum $; P P=$ poids de paille à la récolte $(0 \%$ d'humidité) ; QNR = azote absorbé récolte $(\mathrm{kg} / \mathrm{ha})$.

fait des difficultés rencontrées pour dater précisément ce stade en parcelles d'agriculteurs. Pour caractériser la phase levée-épiaison, on utilisera la relation entre nombre de pieds et nombre d'épis (relation $2 b$ ) malgré sa fiabilité statistique limitée.

On a par ailleurs étudié [étape ii)] l'effet spécifique de l'alimentation azotée et de l'alimentation hydrique sur le comportement du même cultivar, en faisant varier, pour chaque année climatique, les doses d'azote apportées dans les 2 types de sols les plus représentés de la région (sol Tell et sol Hamri, limoneux à sablolimoneux, pouvant présenter une croûte calcaire à une profondeur variable). On a classé les résultats par rapport aux niveaux potentiels des composantes : en année pluvieuse, on atteint en sol Tell ces niveaux potentiels à partir de 60 unités d'azote apportées alors qu'on plafonne à environ 3 quarts du potentiel en sol Hamri ; en année sèche, il n'y a que peu d'effet d'un apport d'azote dans les 2 types de sols et les niveaux des composantes restent limités.

\section{Les parcelles retenues et le protocole d'observations}

Chaque année culturale, les parcelles ont été choisies en variété Karim pour représenter les 2 types de sol Tell et Hamri et explorer, à partir de déclarations a priori des agriculteurs, la variabilité des techniques cul- turales. En première année, on n'a retenu que des parcelles derrière jachère (précédent le plus courant du blé dur) et élargi aux Légumineuses la gamme des précédents culturaux en seconde année. Dix parcelles ont ainsi été étudiées en 1984-1985 et 6 l'année suivante (tableau II) : elles représentent une variabilité de dates et modes de semis ainsi que de niveaux d'intrants explorant la gamme de techniques utilisées dans la région (Besse et Sebillotte, 1991) ; en 19851986, 5 parcelles initialement retenues en sol Tell ont dû être abandonnées car les agriculteurs ne les ont pas menées jusqu'à la récolte du fait de la sécheresse.

Chaque parcelle est représentée par une station homogène vis-à-vis du type de sol, qui constitue l'unité sur laquelle le diagnostic va porter. Sur ces stations, 2 séries de prélèvements et d'observations, au début de la montaison et à la récolte, ont été effectuées. Au sein de chaque station, on a délimité des placettes de 3 rangs sur $50 \mathrm{~cm}$ (soit environ $0,25 \mathrm{~m}^{2}$ de surface unitaire) pour les parcelles semées en ligne et de $50 \mathrm{~cm} x$ $50 \mathrm{~cm}$ pour les parcelles semées à la volée, placettes qui constituent des répétitions. Sur 10 placettes par station et par date de prélèvement, on a mesuré au début de la montaison le nombre de pieds levés et la biomasse aérienne puis, à la récolte, le nombre de pieds, le nombre d'épis, le poids de paille, le nombre de grains et le poids moyen d'un grain. À ces 2 dates (la première étant toujours située avant l'éventuel désherbage chimique), les adventices ont été identifiées et leur matière sèche pesée par placette sauf 


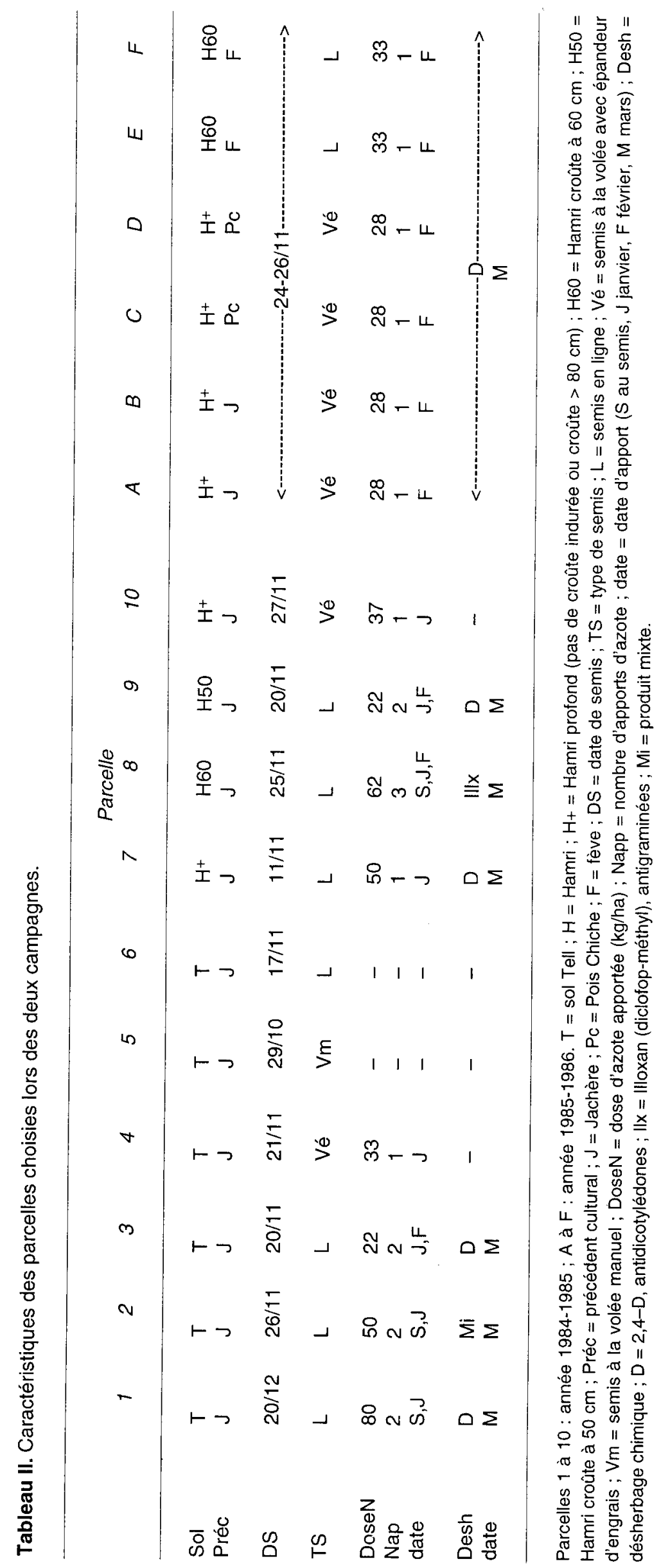


pour le prélèvement de début montaison en 1984-1985 où une seule mesure moyenne a été effectuée par station. En cours de tallage, on a compté les nombres de feuilles à 2 dates successives et déterminé la profondeur de semis sur 75 à 100 pieds par station. Au début de la montaison, à la floraison et à la récolte, on a noté l'éventuelle présence de dégâts d'origine fongique ou parasitaire sur les placettes.

Les autres appréciations des états du milieu ont été réalisées à l'échelle de la station. Une caractérisation de la nutrition azotée par la mesure de l'azote total absorbé à la récolte a été effectuée (teneurs en azote des pailles et des grains, méthode Kjeldhal, 3 échantillons par station issus de l'ensemble des 10 placettes prélevées à la récolte) ainsi qu'une mesure de la teneur en azote des parties aériennes du blé au début de la montaison (même protocole) en 1984-1985. L'appréciation de l'alimentation hydrique comporte par parcelle le calcul d'un bilan hydrique «P-ETP+RU», au pas de temps décadaire ( $P$ pluviométrie, ETP évapotranspiration potentielle, RU réserve utile, exprimées en $\mathrm{mm}$ ). La réserve utile est estimée après mesure de la profondeur d'enracinement à la floraison et par la texture du sol appréciée par analyse granulométrique. Les valeurs d'ETP sont calculées par la formule de Penmann, avec les valeurs d'enracinement à la floraison et par la texture du sol appréciée par analyse de rayonnement de la station de Tunis, située à $60 \mathrm{~km}$ de la zone étudiée, les autres valeurs provenant de la station du Fahs située au sein de la zone.

Une observation de l'état structural de la couche labourée sur chaque station a été effectuée à la floraison ainsi qu'une notation de la profondeur maximale d'enracinement. On a par ailleurs caractérisé les états structuraux des lits de semence à la levée en 1985-1986.

Les itinéraires techniques des agriculteurs ont été recueillis depuis le premier travail effectué sur jachère ou après la récolte du précédent cultural. Les doses de semis enregistrées sont peu variables entre agriculteurs et entre années (de 100 à $120 \mathrm{~kg} / \mathrm{ha}$ ) mais les densités de grains réellement semés sont imprécises car les agriculteurs méconnaissent généralement les poids moyens d'un grain des semences utilisées.

\section{Méthode de traitement des résultats}

La comparaison des valeurs réelles des composantes aux valeurs de référence se fait en calculant par placette le rapport $C_{j} / C_{i p o t}$ (Manichon et al, 1989) appelé degré de réalisation du potentiel : $C_{i}$ est la valeur mesurée de la composante $i$ et $C_{i p o t}$ la valeur maximale que peut prendre $C_{i}$ compte tenu de la valeur de la composante antérieure dans le cycle, $\mathrm{C}_{\mathrm{i}}-1$ (valeur donnée par l'équation $C_{i}=f\left(C_{i-1}\right)$ du tableau $\left.\mathrm{l}\right)$. Par parcelle, on calcule ensuite le degré moyen de réalisation du potentiel (DRP) et son écart type. Pour les relations 4,5 et 6 du tableau I, on procède par comparaison statistique (au seuil de $5 \%$ ) des pentes des droites de régression obtenues expérimentalement d'une part et sur les parcelles d'enquête d'autre part. La placette est prise comme unité de base lorsque cela est possible (relations 4 et 5 ).

\section{RÉSULTATS}

\section{Le rendement et les composantes du rendement}

Au sein de chaque scénario climatique, les rendements obtenus varient beaucoup entre parcelles (tableau III). En 1984-1985, cette variation est très forte en sol Tell (de 10 à plus de 65 $\mathrm{q} / \mathrm{ha}$ ), les rendements étant plus faibles et moins variables en sol Hamri (de 20 à $30 \mathrm{q} / \mathrm{ha}$ ). En 1985-1986, les niveaux de rendement varient fortement en sol Hamri (de 7 à 26 q/ha) mais peuvent atteindre des valeurs proches de celles obtenues lors de l'année pluvieuse (parcelle A).

Les variations de rendement $(R)$ sont, chaque année, étroitement reliées à celles du nombre de grains (NG). En 1984-1985, la pente de la droite de régression $\left(R=4,42{ }^{*} 10^{-3}{ }^{*} \mathrm{NG}, r^{2}=0,95, n\right.$ = 96) n'est pas significativement différente (au seuil de $5 \%$ ) de celle observée dans les situations expérimentales (relation 4 du tableau I). En 1985-1986, la pente de cette droite $(r=3,64$ * $10^{-3}{ }^{*} \mathrm{NG}, r^{2}=0,91, n=58$ ) est statistiquement différente de celle obtenue en conditions expérimentales d'une part, et de celle obtenue sur les parcelles de l'année précédente d'autre part : ceci traduit que le poids moyen d'un grain est inférieur à celui obtenu en 1984-1985. Malgré des différences significatives (au seuil de $5 \%$ ) de la composante poids moyen d'un grain entre parcelles lors de l'année sèche, les variations de rendement se sont essentiellement créées entre semis et fécondation, comme en année pluvieuse. Ces variations proviennent des conditions de croissance du semis à la floraison et non d'éventuels accidents survenus à la fécondation : en effet, on retrouve une forte corrélation entre le nombre de grains $(N G)$ et le poids de paille $(P P)$ à la récolte $\left(N G=15,5^{*} \mathrm{PP}, r^{2}=0,86, n=96\right.$ pour 1984-1985; $N G=16,03{ }^{*} P P, r^{2}=0,88, n=$ 58 pour $1985-1986$ ), les pentes n'étant pas statistiquement différentes entre elles ni différentes de celle obtenue dans les essais (relation 5 du tableau I). L'absence probable d'accidents à la fécondation est confortée par le fait que, chaque année, les températures sont restées dans la gamme $\left(10^{\circ} \mathrm{C}-32^{\circ} \mathrm{C}\right)$ au sein de laquelle la fécondation peut être considérée comme non affectée (Evans et al, 1976 ; Tashiro et Wardlaw, 1990). S'il y a eu un défaut d'alimentation hydrique au cours de cette phase en seconde année, son impact sur le taux de fécondation est resté limité. 
Tableau III. Rendement et composantes du rendement.

\begin{tabular}{|c|c|c|c|c|c|c|}
\hline Parcelle & $\begin{array}{c}R \\
(q / h a)\end{array}$ & $\begin{array}{l}P 1 G \\
(m g)\end{array}$ & $N G$ & $\begin{array}{l}P P \\
(g)\end{array}$ & $N E$ & $N P$ \\
\hline 1 & $\begin{array}{l}56,4 \\
(6,4)\end{array}$ & $\begin{array}{l}44,1 \\
(4,0)\end{array}$ & $\begin{array}{l}12941 \\
(2047)\end{array}$ & $\begin{array}{r}707 \\
(87)\end{array}$ & $\begin{array}{l}334 \\
(44)\end{array}$ & $\begin{array}{l}190 \\
(13)\end{array}$ \\
\hline 2 & $\begin{array}{c}66,2 \\
(12,0)\end{array}$ & $\begin{array}{l}43,1 \\
(2,9)\end{array}$ & $\begin{array}{l}15360 \\
(2454)\end{array}$ & $\begin{array}{r}933 \\
(129)\end{array}$ & $\begin{array}{l}409 \\
(92)\end{array}$ & $\begin{array}{l}319 \\
(38)\end{array}$ \\
\hline 3 & $\begin{array}{r}41,6 \\
(10,9)\end{array}$ & $\begin{array}{l}44,3 \\
(2,3)\end{array}$ & $\begin{array}{c}9432 \\
(2665)\end{array}$ & $\begin{array}{r}603 \\
(140)\end{array}$ & $\begin{array}{l}287 \\
(67)\end{array}$ & $\begin{array}{l}216 \\
(19)\end{array}$ \\
\hline 4 & $\begin{array}{r}25,4 \\
(11,6)\end{array}$ & $\begin{array}{l}43,3 \\
(1,3)\end{array}$ & $\begin{array}{c}4541 \\
(2533)\end{array}$ & $\begin{array}{c}409 \\
(222)\end{array}$ & $\begin{array}{l}170 \\
(72)\end{array}$ & $\begin{array}{l}139 \\
(47)\end{array}$ \\
\hline 5 & $\begin{array}{l}19,1 \\
(5,2)\end{array}$ & $\begin{array}{l}51,4 \\
(3,5)\end{array}$ & $\begin{array}{c}3689 \\
(1066)\end{array}$ & $\begin{array}{r}351 \\
(114)\end{array}$ & $\begin{array}{l}145 \\
(46)\end{array}$ & $\begin{array}{c}88 \\
(39)\end{array}$ \\
\hline 6 & $\begin{array}{l}10,3 \\
(3,2)\end{array}$ & $\begin{array}{l}39,0 \\
(4,2)\end{array}$ & $\begin{array}{c}2620 \\
(781)\end{array}$ & $\begin{array}{r}160 \\
(45)\end{array}$ & $\begin{array}{l}109 \\
20)\end{array}$ & $\begin{array}{l}158 \\
(14)\end{array}$ \\
\hline 7 & $\begin{array}{l}20,1 \\
(7,2)\end{array}$ & $\begin{array}{l}44,4 \\
(4,2)\end{array}$ & $\begin{array}{c}4440 \\
(1196)\end{array}$ & $\begin{array}{l}280 \\
(94)\end{array}$ & $\begin{array}{r}266 \\
(47)\end{array}$ & $\begin{array}{l}235 \\
(50)\end{array}$ \\
\hline 8 & $\begin{array}{l}24,3 \\
(7,8)\end{array}$ & $\begin{array}{l}48,7 \\
(4,4)\end{array}$ & $\begin{array}{c}4921 \\
(1265)\end{array}$ & $\begin{array}{r}329 \\
(138)\end{array}$ & $\begin{array}{r}190 \\
(61)\end{array}$ & $\begin{array}{l}153 \\
(11)\end{array}$ \\
\hline 9 & $\begin{array}{c}30,1 \\
(11,1)\end{array}$ & $\begin{array}{l}47,4 \\
(1,3)\end{array}$ & $\begin{array}{c}6340 \\
(2325)\end{array}$ & $\begin{array}{r}456 \\
(189)\end{array}$ & $\begin{array}{l}206 \\
(90)\end{array}$ & $\begin{array}{l}210 \\
(13)\end{array}$ \\
\hline 10 & $\begin{array}{l}22,5 \\
(8,5)\end{array}$ & $\begin{array}{l}45,2 \\
(2,1)\end{array}$ & $\begin{array}{c}5016 \\
(1945)\end{array}$ & $\begin{array}{r}323 \\
(130)\end{array}$ & $\begin{array}{r}133 \\
(53)\end{array}$ & $\begin{array}{c}58 \\
(19)\end{array}$ \\
\hline$A$ & $\begin{array}{l}25,6 \\
(6,1)\end{array}$ & $\begin{array}{l}36,7 \\
(2,5)\end{array}$ & $\begin{array}{r}6925 \\
(1372)\end{array}$ & $\begin{array}{l}397 \\
(79)\end{array}$ & $\begin{array}{l}276 \\
(46)\end{array}$ & $\begin{array}{l}231 \\
(39)\end{array}$ \\
\hline B & $\begin{array}{l}19,3 \\
(5,2)\end{array}$ & $\begin{array}{l}39,2 \\
(2,3)\end{array}$ & $\begin{array}{c}5006 \\
(1460)\end{array}$ & $\begin{array}{r}290 \\
(76)\end{array}$ & $\begin{array}{r}150 \\
(35)\end{array}$ & $\begin{array}{l}86 \\
\text { (3) }\end{array}$ \\
\hline $\mathrm{C}$ & $\begin{array}{l}15,6 \\
(5,1)\end{array}$ & $\begin{array}{l}33,5 \\
(2,1)\end{array}$ & $\begin{array}{c}4632 \\
(1471)\end{array}$ & $\begin{array}{l}281 \\
(86)\end{array}$ & $\begin{array}{r}190 \\
(58)\end{array}$ & $\begin{array}{r}139 \\
(7)\end{array}$ \\
\hline$D$ & $\begin{array}{r}8,9 \\
(5,5)\end{array}$ & $\begin{array}{l}43,2 \\
(3,1)\end{array}$ & $\begin{array}{c}2042 \\
(1265)\end{array}$ & $\begin{array}{r}126 \\
(74)\end{array}$ & $\begin{array}{c}76 \\
(36)\end{array}$ & $\begin{array}{c}46 \\
(18)\end{array}$ \\
\hline$E$ & $\begin{array}{c}7,1 \\
(1,3)\end{array}$ & $\begin{array}{l}29,7 \\
(3,2)\end{array}$ & $\begin{array}{l}2400 \\
(464)\end{array}$ & $\begin{array}{r}186 \\
(51)\end{array}$ & $\begin{array}{r}119 \\
(25)\end{array}$ & $\begin{array}{l}104 \\
(25)\end{array}$ \\
\hline $\mathrm{F}$ & $\begin{array}{r}8,6 \\
(1,7)\end{array}$ & $\begin{array}{l}31,2 \\
(2,1)\end{array}$ & $\begin{array}{l}2786 \\
(655)\end{array}$ & $\begin{array}{r}205 \\
(59)\end{array}$ & $\begin{array}{r}139 \\
(18)\end{array}$ & $\begin{array}{l}140 \\
(12)\end{array}$ \\
\hline
\end{tabular}

Moyenne sur les 10 placettes de la station; entre parenthèses : écart-type. $R=$ rendement à $0 \%$ d'humidité ; $P 1 G=$ poids moyen d'un grain ; $N G=$ nombre de grains par $m^{2} ; N E=$ nombre d'épis par $m^{2} ; P P=$ poids de paille par $m^{2}$ à la récolte (à $0 \%$ d'humidité) ; $N P=$ nombre de pieds par $\mathrm{m}^{2}$ (récolte). 
La composante nombre de pieds par $\mathrm{m}^{2}$ (NP) varie fortement entre parcelles chaque année (de 58 à 319 pieds $/ \mathrm{m}^{2}$ en $1984-1985$; de 46 à 230 pieds $/ \mathrm{m}^{2}$ en 1985-1986). Elle est souvent située dans la gamme $\left(N P<160\right.$ pieds $/ \mathrm{m}^{2}$, cf relation 1 du tableau I) où elle peut être considérée comme limitant directement le niveau de biomasse en début de montaison (parcelles $4,5,6,8$ et 10 en 1984-1985 ; toutes parcelles sauf A en 19851986). Par ailleurs, chaque année, certaines parcelles présentent une très forte variabilité interne de cette composante (coefficients de variation supérieurs à $30 \%$ pour les parcelles $4,5,10$ en 1984-1985 et pour D en 1985-1986).

On note une forte variabilité entre parcelles (fig 1) du degré moyen de réalisation du potentiel d'épis (DRPE) et du degré moyen de réalisation du potentiel de grains (DRPG) : seules 2 parcelles, lors de la première année, présentent des nombres d'épis et de grains proches du potentiel. Par ailleurs, les valeurs des 2 rapports sont très voisines au sein d'une parcelle, et ce pour la grande majorité d'entre elles.

Le tableau IV récapitule pour les 2 scénarios climatiques les étapes de l'élaboration du rendement par parcelle.

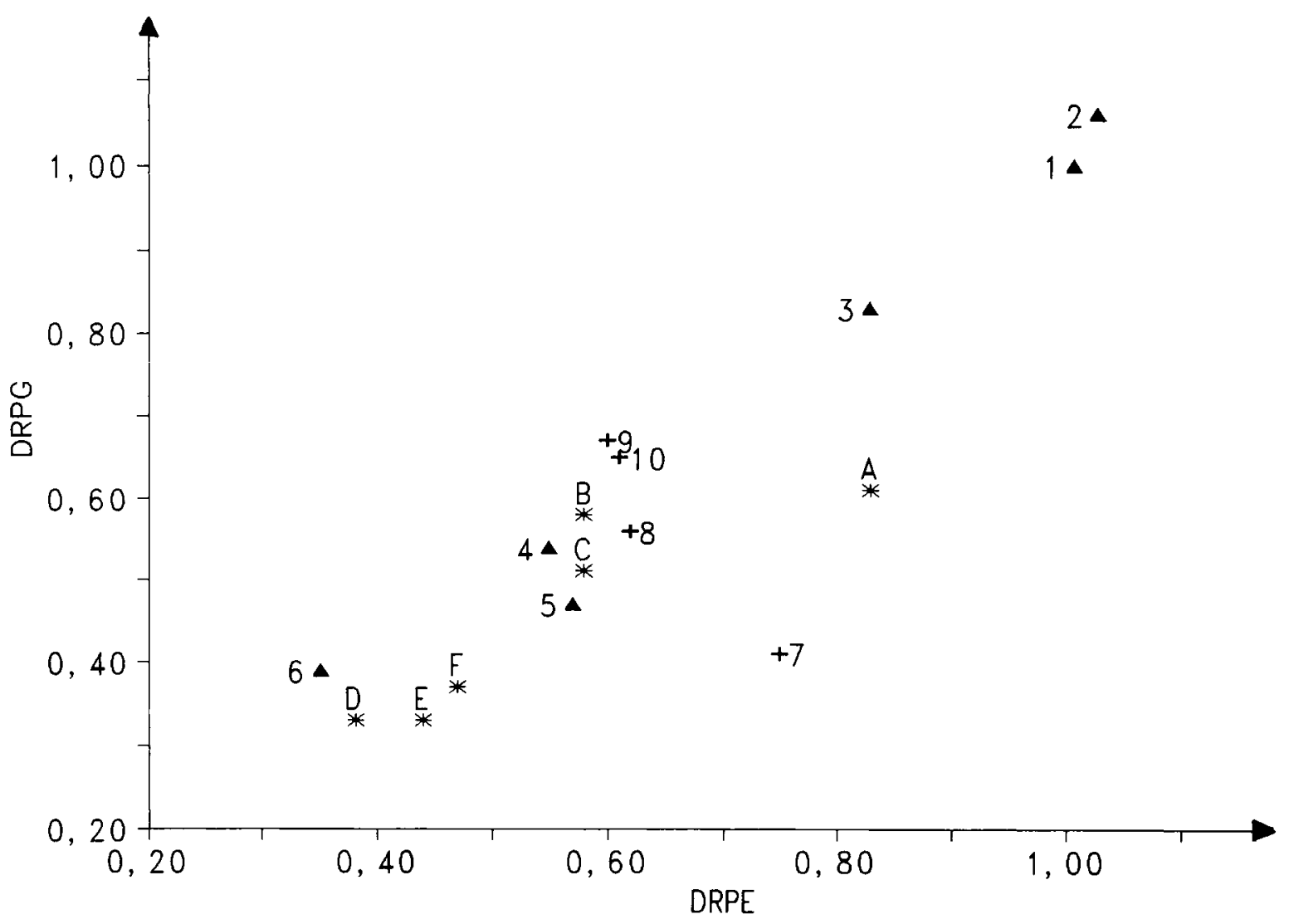

\section{Facteurs et conditions limitants au cours des phases successives d'élaboration du rendement}

L'observation des états du milieu et du peuplement renseigne sur les facteurs et conditions intervenant sur le niveau des composantes du rendement (tableau V).

\section{Élaboration du nombre de pieds}

En 1984-1985, les plus faibles nombres de pieds et les plus fortes variabilités intraparcellaires de cette composante sont reliées à la pratique du semis à la volée, quelle qu'en soit la date (parcelles $4,5,10)$.

En 1985-1986, on obtient des nombres de pieds très variables, notamment pour les semis à la volée (parcelles $A, B, C$ et $D$ ), alors que les dates de semis (fin novembre, soit à la fin des séquences pluvieuses d'automne) et les densités semées sont identiques : on constate que les dates de levée déduites des distributions de nombre de feuilles à la fin février (fig 2) diffèrent entre parcelles (pour $75 \%$ des pieds observés, autour du 20 décembre en $A$ et $C$, du 6-8 janvier 
Tableau IV. Récapitulatif de l'élaboration du rendement dans les parcelles.

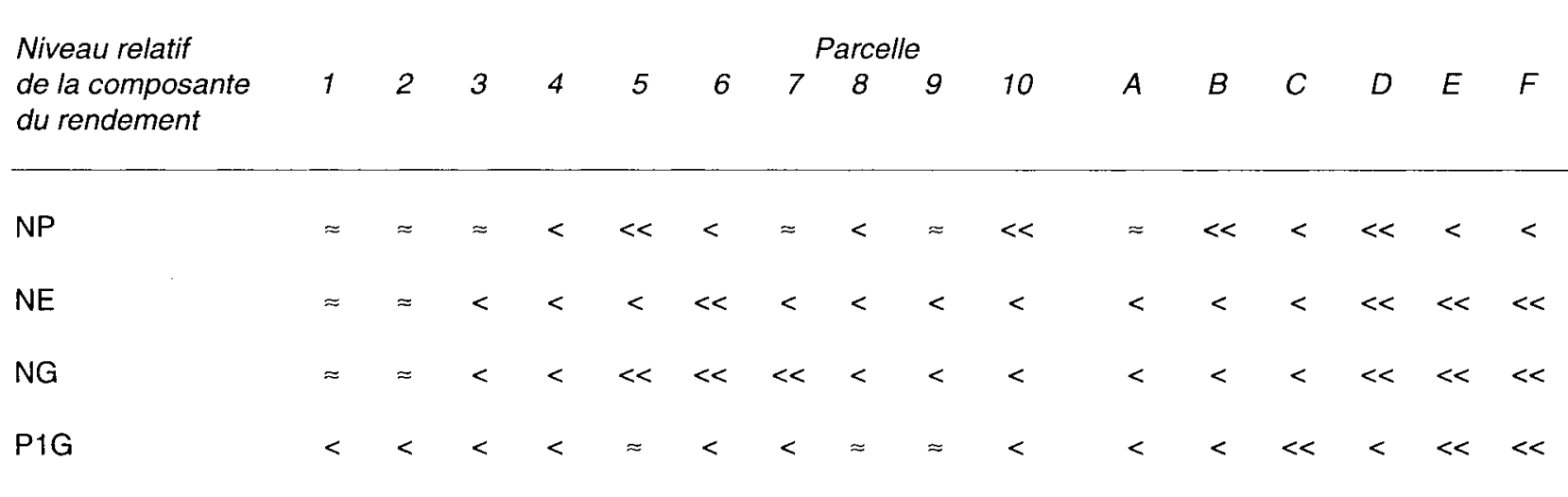

$\mathrm{NP}=$ nombre de pieds $/ \mathrm{m} 2 ; \mathrm{NE}=$ nombre d'épis $/ \mathrm{m}^{2} ; \mathrm{NG}=$ nombre de grains $/ \mathrm{m}^{2} ; \mathrm{P} 1 \mathrm{G}=$ poids moyen d'un grain. Pour $\mathrm{NP}:$ utilisation de la relation 1 du tableau $\mathrm{I}$. équivaut à $\mathrm{NP} \geq 160$ pieds $/ \mathrm{m}^{2} ;<$ à $100<\mathrm{NP}<160$ pieds $/ \mathrm{m}^{2} ;<<$ à $\mathrm{NP}<100$ pieds $/ \mathrm{m}^{2}$. pour NE et $N G$ : utilisation du degré de réalisation du potentiel (DRPE et DRPG). $\approx$ équivaut à DRP $\geq 90 \% ;<$ à $(50 \% \leq \mathrm{DRP}<90 \%) ;<<$ à $(\mathrm{DRP}$ $<50 \%$ ). Pour $\mathrm{p} 1 \mathrm{G}$ : utilisation de la relation 4 du tableau $\mathrm{I}$. $\approx$ équivaut à $(\mathrm{P} 1 \mathrm{G} \geq 46,3 \mathrm{mg}) ;<$ à $(35 \leq \mathrm{P} 1 \mathrm{G}<46,3 \mathrm{mg}) ;<<$ à $(\mathrm{P} 1 \mathrm{G}<35$ $\mathrm{mg}$.

Tableau V. États du milieu et du peuplement dans les parcelles.

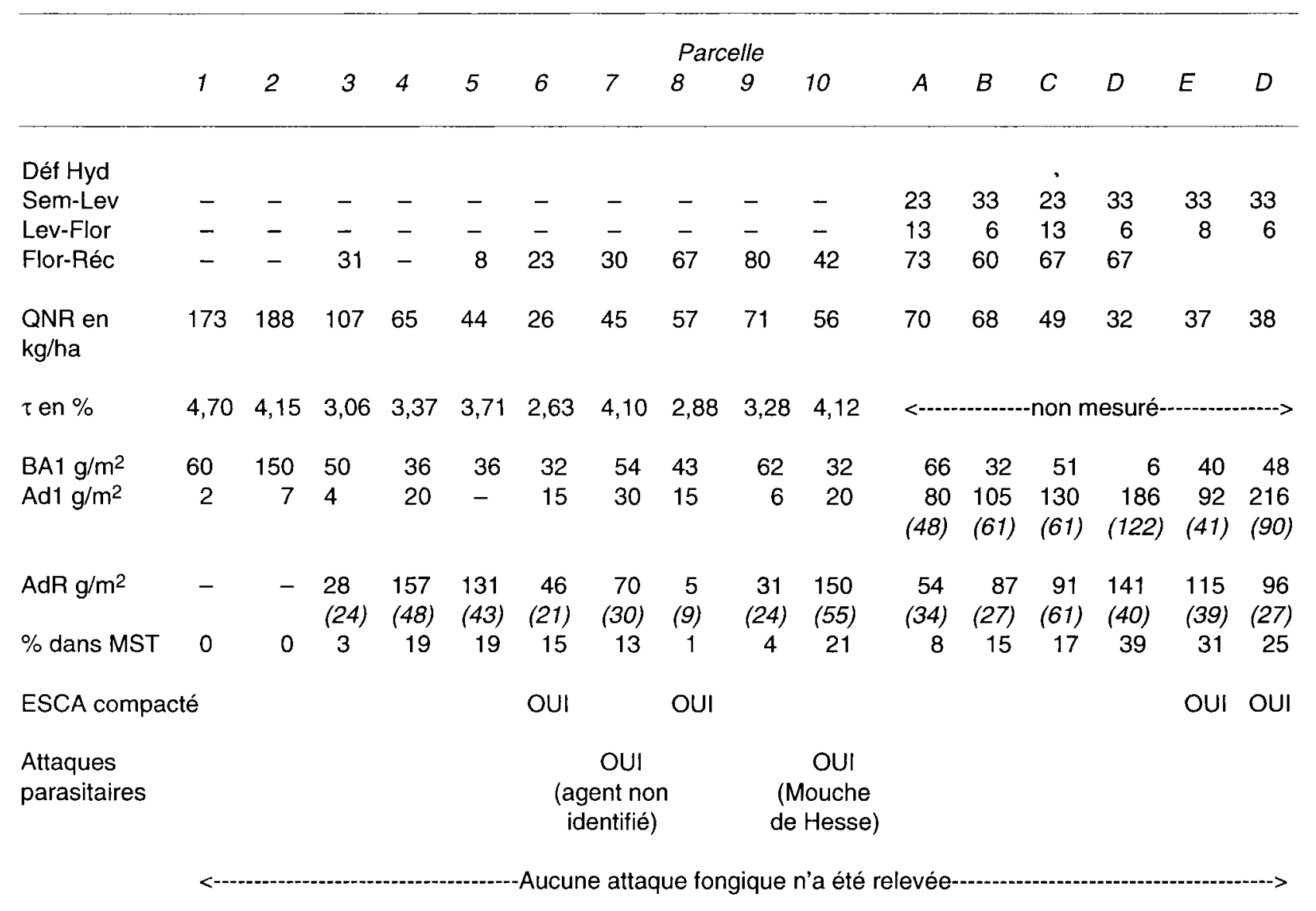

Def Hyd = déficit hydrique cumulé sur la période $(\mathrm{mm}) ;$ Sem $=$ Semis $;$ Lev = Levée $;$ Flo = Floraison $;$ Réc = Récolte $;$ QNR = azote absorbé à la récolte par les parties aériennes ; $t=$ teneur en azote en début de montaison; $B A 1$ = biomasse aérienne en début de

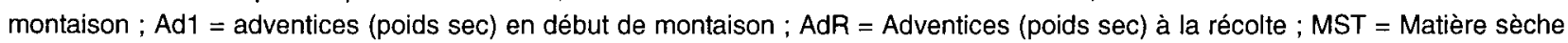
totale à la récolte (parties aériennes du blé $+\mathrm{AdR}$ ) ; ESCA = État structural de la couche arable. Les valeurs entre parenthèses correspondent aux écarts-types. 

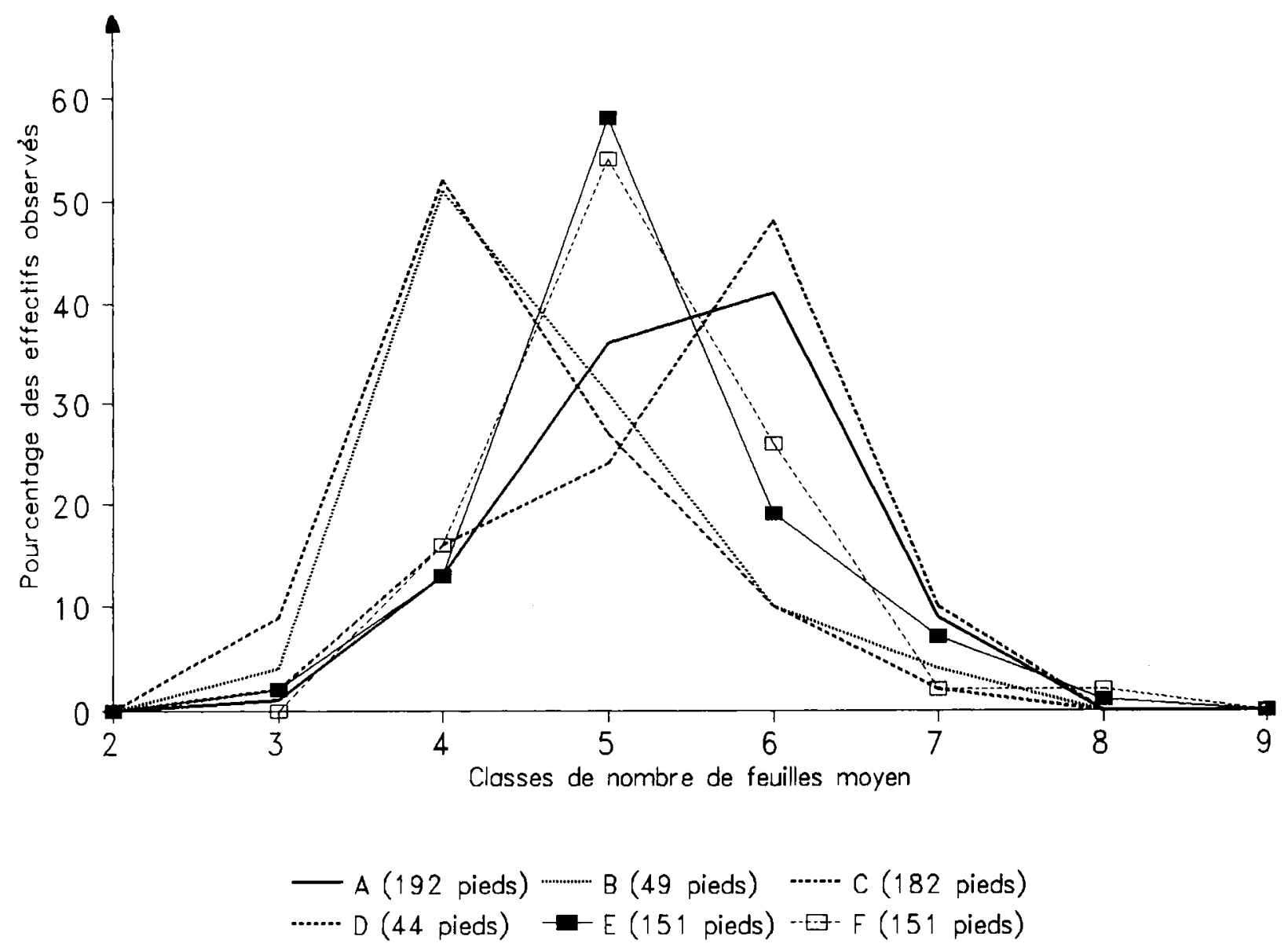

Fig 2. Nombre de feuilles par pied en 1985-1986 (27/2/86). Les classes indiquées correspondent au nombre de feuilles inférieur (ex : classe 5 feuilles $=$ de 5 feuilles inclus à 6 feuilles exclues).

pour $E$ et $F$, du 15 au 20 janvier pour $B$ et $D$ ) et que les plus faibles nombres de pieds correspondent aux dates de levée les plus tardives (parcelles $B$ et $D$ ). Lors de cette campagne culturale, les lits de semence sont tous constitués de terre fine et de petites mottes (a priori non défavorables aux levées) mais montrent des différences d'épaisseur de la couche travaillée par les outils de préparation superficielle du sol $(8$ à $10 \mathrm{~cm}$ en $A, C, E$ et $F ; 15$ à $18 \mathrm{~cm}$ en $B$ et D). La profondeur de semis des semences ayant émergé est faible et homogène pour les semis en ligne ( $E$ et F), plus variable pour les semis à la volée, tous suivis d'un passage de covercrop enfouissant les semences de façon hétérogène (fig 3 ) : en $A$ et $C$, les semences levées sont ainsi en majorité situées à proximité du fond du travail superficiel alors qu'en $B$ et $D$ (comme en $E$ et F) elles sont éloignées de cette discontinuité. Les semis ayant eu lieu dans des profils réhumectés par les pluies d'automne mais soumis par la suite à forte dessiccation, les semences se trouvaient dans un environnement asséché et n'ont pu germer que lors du retour des pluies ( $E$ et $F$ ), sauf si, placées à proximité du fond du travail superficiel, elles ont pu le faire à la faveur de l'humidité résiduelle ( $A$ et $C)$; en $B$ et $D$, les semences situées près de cette discontinuité ont probablement pu germer mais non émerger compte tenu de leur profondeur. En 1984-1985, la régularité des pluies après semis a probablement partiellement masqué la variabilité de fonctionnement des lits de semence en homogénéisant les profils hydriques.

\section{Élaboration du nombre d'épis et du nombre de grains}

En 1984-1985, on constate dès le début de la montaison de fortes différences de teneur en azote des parties aériennes $(\tau)$, certaines parcelles seulement $(1,2,7,10)$ s'approchant de la teneur de l'ordre de $4,5 \%( \pm 0,8 \%)$ considérée par Meynard (1985b) comme témoignant d'une nutrition azotée précoce non limitante. Ces teneurs (fig 4) ne sont que pour partie reliées aux degrés de réalisation du potentiel d'épis DRPE 

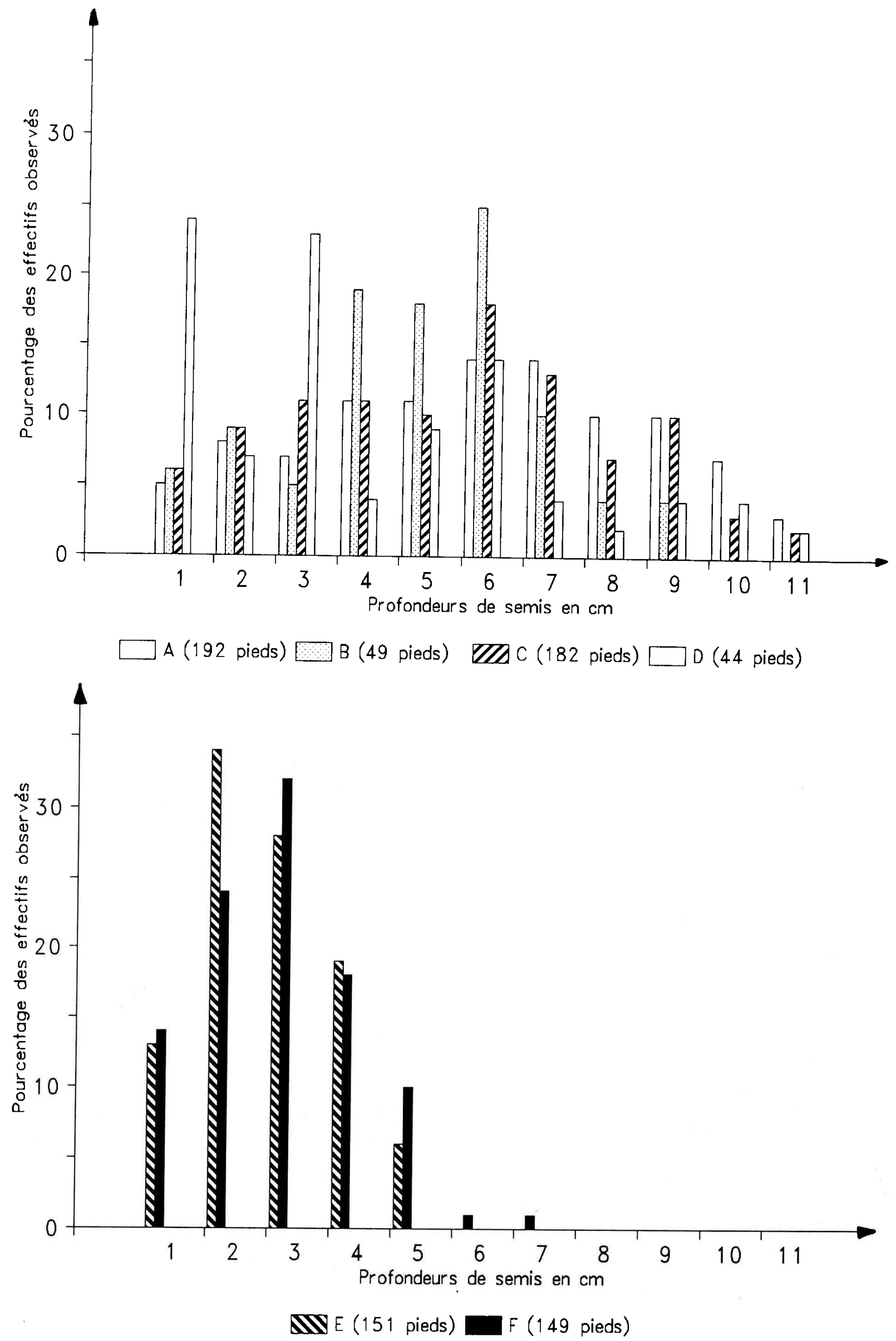

Fig 3. Profondeurs de semis des semences ayant levé dans les parcelles de 1985-1986. a. Parcelles semées à la volée. b. Parcelles semées en ligne. 


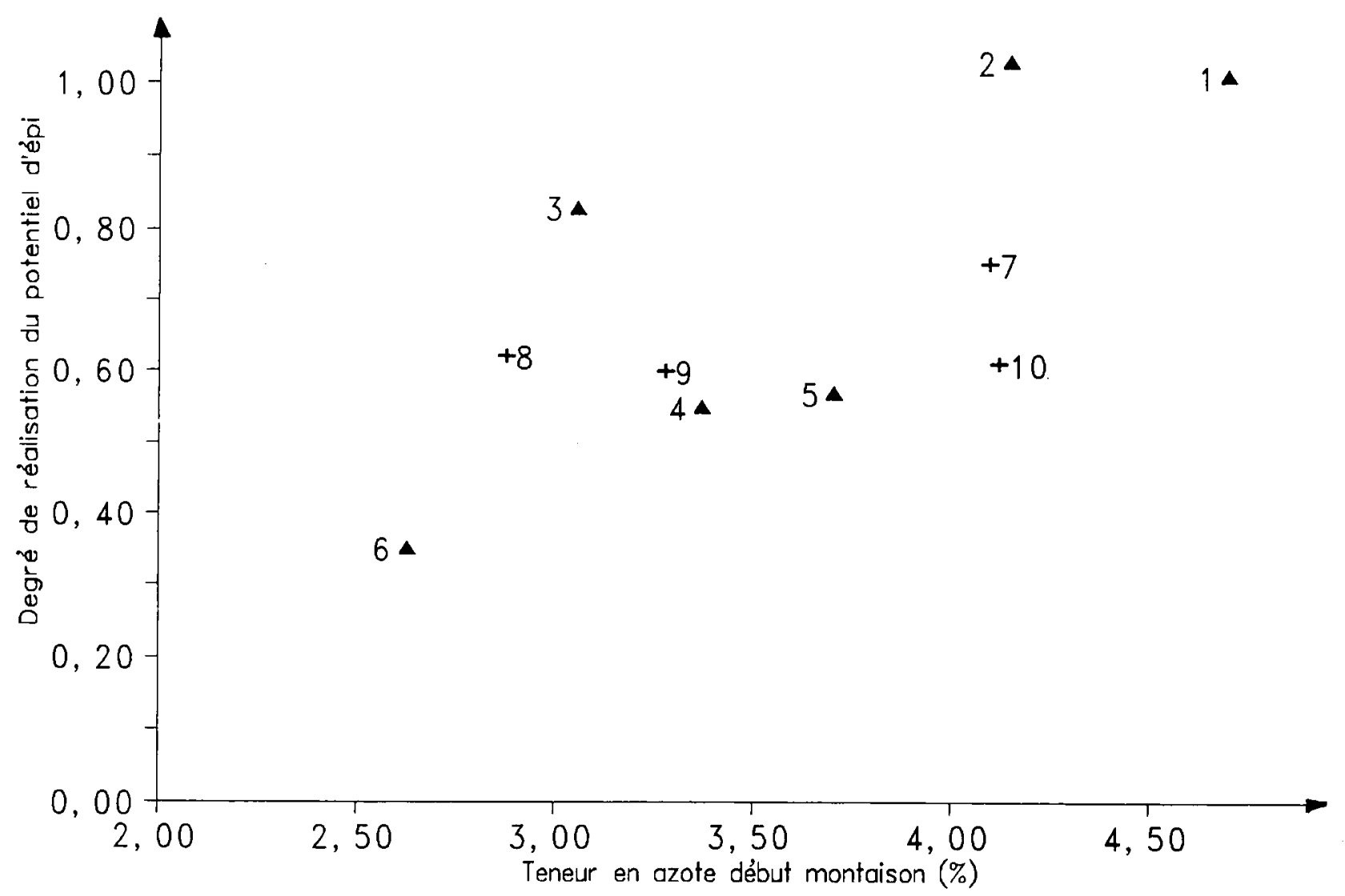

Fig 4. Relation entre la teneur en azote des parties aériennes en début de montaison et le degré de réalisation du potentiel d'épis en 1984-1985. A Tell ; + Hamri.

(DRPE $\left.=0,223^{\star} \tau-0,109, r^{2}=0,48, n=10\right)$ : d'autres facteurs limitants que le statut azotée précoce sont intervenus dans l'élaboration du nombre d'épis dans certaines parcelles. Par ailleurs, en 1984-1985, l'absorption d'azote dans les parties aériennes sur l'ensemble du cycle (QNR) est fortement reliée au nombre de grains obtenus (QNR $=12,2^{*} N G^{*} 10^{-3}, r^{2}=0,98, n=$ 10), la pente n'étant pas significativement différente de celle obtenue expérimentalement (relation $6 \mathrm{du}$ tableau I). En 1985-1986, la gamme d'absorption d'azote est limitée et la corrélation avec le nombre de grains est moindre (QNR = $11,8^{\star} N G^{\star} 10^{-3}, r^{2}=0,62, n=6$ ), la pente étant significativement plus faible que celle établie en conditions expérimentales d'une part, et en parcelles agricoles en 1984-1985 d'autre part. Ceci traduit une moins bonne valorisation de l'azote absorbé en termes de nombre de grains formés. Par ailleurs, les indices de récolte d'azote (quantité d'azote contenue dans les grains rapportée à l'azote total contenu dans les parties aériennes à la récolte) sont compris entre 75 et $80 \%$, sauf pour les parcelles $E$ et $F$ (57 et $62 \%$ respectivement) dont les teneurs en azote des pailles à la récolte sont particulièrement fortes $(0,85$ et
$0,71 \%$ respectivement contre 0,35 à $0,45 \%$ pour les autres parcelles).

Des infestations élevées en adventices ont été constatées dès le début de la montaison, dans plusieurs parcelles en 1984-1985 et dans toutes en 1985-1986. Elles sont majoritairement constituées de ray-grass, avec, localement, des dicotylédones dont des pieds de millepertuis entraînant de fortes variations intraparcellaires des matières sèches d'adventices. Chaque année, les plus forts taux pondéraux d'adventices à la récolte correspondent aux plus faibles DRPG mais une caractérisation plus fine des infestations aurait été nécessaire pour apprécier leur impact réel sur la période levée-floraison. La forte différence de degrés de réalisation des nombres d'épis et des nombres de grains en parcelle 7 est vraisemblablement due à l'attaque parasitaire observée ; celle qui concerne la parcelle $A$ reste inexpliquée.

\section{Alimentation hydrique et poids d'un grain}

Lors de la campagne 1984-1985, les déficits hydriques calculés pendant la période de rem- 
plissage des grains ont été tardifs et d'ampleur limitée, sauf pour 2 parcelles. En 1985-1986, des déficits hydriques ont été enregistrés à plusieurs phases du cycle dans toutes les parcelles et les réserves utiles n'ont été que très rarement remplies ( 1 ou 2 décades pendant le mois de mars). Pour chaque année, les variations de poids moyen d'un grain sont sans relation avec les déficits hydriques calculés, leur différence entre années pouvant être attribuée à la différence d'alimentation hydrique et/ou à l'existence de températures élevées en $1985-1986$ ( $>35^{\circ} \mathrm{C}$ dans la dernière décade de mai), notamment pour les parcelles à floraison tardive (B et $D)$.

On a noté par ailleurs dans certaines parcelles des états structuraux compacts de la couche arable (parcelles 6, 8, E et F).

\section{DISCUSSION}

On a retrouvé, au cours de ces 2 campagnes culturales et dans cette région semi-aride, une forte variabilité interannuelle des rendements liée à la pluviométrie : en sol Tell par exemple, des rendements de l'ordre de $60 \mathrm{q} / \mathrm{ha}$ ont été enregistrés en parcelles d'agriculteurs la première année et aucune production de grains n'a été obtenue en seconde année. Cependant, on met aussi en évidence l'importance des variations intra-annuelles de production : ainsi, en année climatiquement très favorable comme 1984-1985 où des rendements proches du potentiel peuvent être atteints en conditions agricoles, certaines parcelles obtiennent des niveaux de productions très faibles. A contrario, en année sèche comme 1985-1986, des niveaux considérés comme corrects pour la région (Latiri-Souki et Essamet, 1989) peuvent être atteints chez les agriculteurs. Ces différences de production sont observées sans intervention du facteur variétal.

La réussite de la levée, en taux et en dynamique, apparaît comme un élément à la fois très variable et fondamental dans l'élaboration du rendement lors des 2 scénarios climatiques. On ne peut exclure l'influence d'éventuelles différences de densités réelles de semis ou de pouvoirs germinatifs des semences utilisées. Cependant, le positionnement relatif des semences par rapport aux discontinuités structurales et hydriques dans le profil semble être déterminant : on retrouve en particulier une forte variabilité des résultats en semis à la volée, où le contrôle de ces profondeurs est aléatoire
(Fenech et Papy, 1977 ; Bouaziz et Burckler, 1989).

Le rôle joué par le nombre de pieds est d'autant plus important qu'en situation de peuplement faible on n'a observé sur ces parcelles que très peu de compensation par ramification : les degrés de réalisation du potentiel d'épis correspondent fréquemment à des niveaux de tallageépi proches de 1, très inférieurs à ceux constatés en densités faibles en conditions non limitantes (Latiri-Souki et al, 1992). En outre, la proximité des valeurs des degrés de réalisation des nombres d'épis et de grains dans la majorité des parcelles laisse penser que, au cours de la phase d'élaboration de la composante nombre de grains par $\mathrm{m}^{2}$, l'influence du nombre de pieds levés n'a pu être tempérée à aucun moment.

Cette cohérence dans le fonctionnement des peuplements entre la levée et la floraison suggère, dans les cas où les parcelles sont situées en dessous de leurs potentiels respectifs, qu'un facteur limitant majoritaire ou une même combinaison de facteurs sont intervenus pendant l'ensemble de la période concernée: l'analyce a montré que la nutrition azotée des peuplements pouvait être en cause, certaines conditions défavorables (parasitisme, état structural dégradé) pouvant limiter encore la disponibilité de l'azote pour les peuplements. Mais, surtout, l'effet des adventices, parfois en quantité très importante, peut augmenter cette compétition pour l'azote, ainsi que pour l'eau, surtout en conditions sèches lors de la deuxième année, comme d'autres auteurs l'ont montré en climat semiaride (Cooper et al, 1987 ; McAneney et Arrúe, 1993).

Les relations entre niveaux d'infestation et pratique du désherbage chimique ne sont pas univoques : les plus fortes infestations se rencontrent en 1984-1985 dans les parcelles non désherbées chimiquement et la présence de désherbage chimique (pratiqué, chaque année, en cours de montaison) est reliée à la stagnation ou à la diminution de la matière sèche d'aventices entre début montaison et récolte ; cependant, des niveaux variables d'infestation existent lors des 2 années en présence de désherbage chimique, le plus souvent réalisé avec uniquement un produit antidicotylédones. Le niveau d'infestation de départ, pas plus que l'amplitude des évolutions des infestations en cours de cycle cultural, ne peuvent s'interpréter en l'absence d'informations sur les stocks initiaux et sur les 
dynamiques de levée des adventices. Dans cette région, la rareté de la lutte contre les Graminées adventices correspond souvent pour les agriculteurs à la recherche d'une qualité fourragère suffisante dans la jachère paturée qui suit le blé (Besse et Sebillotte, 1991 ; Latiri-Souki et Aubry, 1991). En outre, nous n'avons pas pu mettre en évidence, même en année régulièrement pluvieuse, de relation entre l'alimentation azotée des peuplements telle qu'elle a été appréciée et la fertilisation azotée : ceci peut s'interpréter comme un effet des autres postes de fournitures d'azote, non mesurés ici mais certainement très variables (Vlek et al, 1981), ainsi que comme un effet probable des peuplements adventices. L'absence de mesure des quantités totales d'azote prélevées par l'ensemble du couvert (blé et adventices) ne permet cependant pas de tester cette hypothèse. II reste qu'on peut difficilement escompter un effet direct notable de la fertilisation azotée sur les rendements en blé, compte tenu de l'ampleur des variations des densités de peuplement cultivé et des infestations adventices.

Par ailleurs, les faibles valeurs de l'indice de récolte d'azote, associées à de fortes concentrations d'azote dans les pailles, traduisent un transfert déficient de cet élément des parties végétatives vers les grains dans la dernière partie du cycle, du fait de conditions hydriques défavorables (Triboï, 1990 ; Gonzalez Ponce et al, 1993 ; Giunta et al, 1993) : ce cas n'a été observé dans notre échantillon qu'en deuxième année pour les parcelles $E$ et $F$, qui présentent des états structuraux compactés de la couche arable défavorables à l'absorption de l'eau et des éléments minéraux.

L'absence d'explication donnée à certaines variations d'élaboration du rendement entre parcelles (variations du poids moyen d'un grain par exemple) est décevante. Elle est pour partie due à l'échantillon réduit de parcelles objets du diagnostic mais aussi à l'absence de certaines mesures (portant notamment sur les nutritions azotée et hydrique) qui auraient permis de mener une analyse plus fine, difficile cependant à mettre en œuvre sur un réseau de parcelles agricoles. Par ailleurs, on peut également voir dans cette limitation le reflet d'une certaine carence des références concernant l'élaboration du rendement du blé dur en climat semi-aride et dans les conditions de la pratique agricole. Ces limites ne nous semblent pas cependant remettre en cause la méthode retenue, face en particulier à l'alternative d'un diagnostic fondé sur une approche corrélative des performances et des techniques : c'est au contraire un accroissement des contrôles sur les états du milieu qui aurait permis d'aller plus loin dans les conclusions.

\section{CONCLUSION}

Dans le cas du blé dur en conditions semi-arides, l'utilisation diagnostique de références expérimentales paramétrées en conditions non limitantes montre son intérêt pour analyser les facteurs et conditions limitant le rendement en parcelles agricoles, comme cela a été illustré sur d'autres cultures et dans d'autres milieux (Le Bail et al, 1990 ; Diouf, 1991 ; Doré, 1992).

Elle comporte cependant des limites qui renvoient en partie à la nécessité de contrôles plus précis du milieu (azote absorbé par les adventices, meilieur suivi hydrique...) mais aussi à la nature même des références expérimentales. Ce dernier point débouche sur la nécessité d'expérimentations complémentaires pour pouvoir améliorer les diagnostics : dans la situation étudiée, il serait souhaitable de mieux connaître le fonctionnement de peuplements de blé dur, dans divers scénarios climatiques, en présence de niveaux d'infestation en adventices tels que nous les avons rencontrés et qui sont en partie constitutifs des systèmes de culture du blé dur dans cette région. La démarche adoptée permet ainsi d'orienter des programmes de recherche et de développement vers des objectifs prioritaires.

Cependant, en l'état, notre étude montre que c'est par un raisonnement tout au long du cycle des interactions entre états du milieu, états du peuplement et techniques que l'on peut espérer augmenter et surtout régulariser la production, plus qu'à travers l'accroissement simple des quantités d'intrants, coûteux à l'échelle globale car rarement efficaces. La prise en compte opérationnelle de ces interactions pour la maîtrise technique de la conduite du blé dur est cependant complexe dans un cadre marqué par une forte incertitude (aléas climatiques, variabilité des effets précédents...) et par des contraintes importantes liées aux systèmes de production (limitations fortes des capacités économiques, imbrication étroite des secteurs d'activité au sein de l'exploitation). 


\section{RÉFÉRENCES}

Besse T, Sebillotte M (1991) Les systèmes de culture céréaliers des exploitations agricoles privées : nature et raisons d'être. In : Pour une approche régionale du développement agricole : céréaliculture et dynamique de systèmes agraires en Tunisie, Ann Inst Natl Rech Agron Tunis, spécial 64, 167187

Bouaziz A, Bruckler L (1989) Modeling of wheat inhibition and germination as influenced by soil physical properties. Soil Sci Soc Am J 53, 1, 219-227

Burleigh JR, Yamoah CF, Regas JL, Eylands VJ (1991) Analysis of factors related to wheat yield on farm fields in the Buberuka Highlands of Rwanda. Agron J 83, 625-631

Collectif (1988) Fonctionnement d'exploitations agricoles de la wilaya de Sidi-Bel-Abbes. In : Projet de recherche/développement sur l'intensification des céréales, légumes secs et fourrages dans la wilaya de Sidi-Bel-Abbes, INRA/ITGC/IAM, 61 p + annexes

Cooper PJM, Gregory PJ, Tully D, Harris HC (1987) Improving water use efficiency of annual crops in the rainfed farming systems of West Asia and North Africa. Exp Agric 23, Farming Systems Series-5, 113-158

Diouf $M$ (1991) Analyse de l'élaboration du rendement du mil (Pennisetum typhoides Stapf et Hubb) : mise au point d'une méthode de diagnostic en parcelles paysannes. Thèse doct INA-PG, Paris, $227 \mathrm{p}+$ annexes

Doré T (1992) Analyse, par voie d'enquête de la variabilité des rendements et des effets précédents du pois protéagineux de printemps (Pisum sativum $L$ ). Thèse doct INA-PG, Paris, $214 p$ + annexes

Durrant MJ (1988) A survey of seedling establishment in sugar beet crops in 1980 and 1981. Ann Appl Biol $113,347-355$

Durrant MJ, Jaggard KW, Scott RK (1984) Meeting the challenge for sugar beet: magnitude and origin of the problem and possible solutions. Ann App/ Biol 7, 85-102

El Amami S, Bouzaidi A (1979) Carte de la vocation céréalière de la Tunisie et estimation de la production annuelle en céréales. Doc roneo Centre de Recherches du Génie Rural, Tunis, 7 p + carte

Evans LT, Wardlaw IF, Fischer RA (1976) Wheat. In: Crop Physiology (LT Evans, ed) Cambridge Univ Press, 101-149

Fenech J, Papy F (1977) Conditions de réussite de la levée en climat méditerranéen. Cas des cultures de céréales en sec au nord du Maroc. Ann Agron 28 (6), 599-635

Giunta F, Motzo R, Deidda M (1993) Effect of drought on yield and yield components of durum wheat and triticale in a Mediterranean environment. Field Crops Research 33, 399-409

Gonzalez Ponce R, Salas ML, Mason SC (1993) Nitrogen use efficiency by winter barley under diffe- rent climatic conditions. J Plant Nutr 16 (7), 12491261

Latiri-Souki K, Aubry C (1991) Les céréales dans le semi-aride : potentialités, variations et contraintes. In : Pour une approche régionale du développement agricole : céréaliculture et dynamique de systèmes agraires en Tunisie. Änn Inst Natl Rech Agron Tunis, Numéro spécial, 64, 189-230

Latiri-Souki K, Aubry C, Doré T, Sebillotte M (1992) Élaboration du rendement du blé dur en conditions semi-arides en Tunisie : relations entre composantes du rendement sous différents régimes de nutrition azotée et hydrique. agronomie 12 (1), 31-43

Latiri-Souki K, Essamet M (1989) Les céréales : variabilité interannuelle, aléa climatique et progrès technique. In : Journées Slah Eddine El Amami, Tunis 30 mai 1989, 42-47

Lelièvre F, El Mourid M, Abdani M (1981) La culture de blé dur en sec dans les Doukkala. Résultats d'une enquête culturale réalisée pour apprécier les contraintes d'alimentation hydrique. Hommes Terres et Eaux, Rabat (Maroc) 42, 35-47

Le Bail M, Meynard JM, Sebillotte M, Delpit B (1990) An agronomic diagnosis for spring barley adapted to brewery. In : First Congr Eur Soc of Agron, Paris, (A Scaife, ed), $2 p$

McAneney KJ, Arrúe JL (1993) A wheat-fallow rotation in northeastern Spain: water balance-yield considerations. agronomie 13, 481-490

Manichon H, Roger-Estrade JR, Leterme P (1989) Analyse intégrée des rendements et de leurs causes de variation entre parcelles. In : Programme "Rotations Céréalières intensives", Convention INRA/ONIC/ITCF, Paris, $71 \mathrm{p}+$ annexes

Meynard JM (1985a) Construction d'itinéraires techniques pour la conduite du blé d'hiver. Thèse DDI, INA-PG, Paris, $297 p$

Meynard JM (1985b) Les besoins azotés du blé d'hiver jusqu'au début de la montaison. agronomie 5 (7), 579-589

Meynard JM, Sebillotte M (1982) Diagnostic sur les causes de variation du rendement du blé dans une petite région. In : La fatigue des sols. Série Les colloques de l'INRA, Paris, 157-168

Meynard JM, David G (1992) Diagnostic de l'élaboration du rendement des cultures. Cah Agric 1, 9-19

Ollivier M, Malet P (1979) Un modèle d'action des techniques culturales sur le blé. I. Principe et premiers résultats sur une variété cultivée dans un domaine expérimental. Ann Agron 30 (6), 529-551

Papy F (1979) Analyse du comportement des cultures de blé et d'orge dans différentes régions céréalières du Maroc à travers leur réaction aux variations interannuelles des régimes pluviométriques. $C R$ Acad Agric Fr 3, 231-247

Sebillotte M (1978) La collecte des références et les progrès de la connaissance agronomique. In : Exigences nouvelles pour l'agriculture. Les systèmes de culture pourront-ils s'adapter? (J Boiffin, $P$ Huet, M Sebillotte, eds), ADEPRINA/INA ParisGrignon, Paris, 466-496 
Sebillotte M (1990) Approaches of the on-farm agronomist: illustrated methodological considerations. Development oriented research on agrarian systems project, Katesart Univ, Thaïlande, 36 p

Sebillotte M, Boiffin J, Caneill J, Meynard JM (1978) Sécheresse et fertilisation azotée du blé d'hiver. Bull AFES 3, 197-214

Shafiq M, Azeem M, Longmire J (1993) Diagnosing alternatives in conventional crop rotations: sunflowers as an alternative to wheat in the cottonbased cropping systems of Pakistan's Punjab. Agric Syst 42, 245-264
Tashiro T, Wardlaw IF (1990) The reponse to high temperature shock and humidity changes prior and during the early stages of grain development in wheat. Aust J Plant Physiol 17, 551-561

Triboï E (1990) L'agrophysiologie de la qualité : effet du milieu climatique et nutritionnel sur l'expression du potentiel génétique de la qualité. In: Glutamines de haut poids moléculaire et qualité des blés, 4 décembre 1990, Paris, 59-76

Vlek PLG, Fillery IRP, Burford JR (1981) Accession, transformation and loss of nitrogen in soils of the arid region. Plant and Soil 58, 97-175 